\title{
Operating at a Distance - How a Teleoperated Surgical Robot Reconfigures Teamwork in the Operating Room
}

\author{
HANNAH R. M. PELIKAN*, University of Twente, The Netherlands \\ AMY CHEATLE, Cornell University, USA \\ MALTE F. JUNG, Cornell University, USA \\ STEVEN J. JACKSON, Cornell University, USA
}

\begin{abstract}
This paper investigates how a teleoperated surgical robot reconfigures teamwork in the operating room by spatially redistributing team members. We report on findings from two years of fieldwork at two hospitals, including interviews and video data. We find that while in non-robotic cases team members huddle together, physically touching, introduction of a surgical robot increases physical and sensory distance between team members. This spatial rearrangement has implications for both cognitive and affective dimensions of collaborative surgical work. Cognitive distance is increased, necessitating new efforts to maintain situation awareness and common ground. Moreover, affective distance is introduced, decreasing sensitivity to shared and non-shared affective states and leading to new practices aimed at restoring affective connection within the team. We describe new forms of physical, cognitive, and affective distance associated with teleoperated robotic surgery, and the effects these have on power distribution, practice, and collaborative experience within the surgical team.
\end{abstract}

CCS Concepts: • Human-centered computing $\rightarrow$ Computer supported cooperative work; Empirical studies in collaborative and social computing; Collaborative and social computing theory, concepts and paradigms;

Additional Key Words and Phrases: distance; teamwork; teleoperation; robotic surgery

ACM Reference Format:

Hannah R. M. Pelikan, Amy Cheatle, Malte F. Jung, and Steven J. Jackson. 2018. Operating at a Distance - How a Teleoperated Surgical Robot Reconfigures Teamwork in the Operating Room. Proc. ACM Hum.-Comput. Interact. 2, CSCW, Article 138 (November 2018), 28 pages. https://doi.org/10.1145/3274407

\section{INTRODUCTION}

Teleoperated robotic systems allow teams to perform work from a distance in areas that are inaccessible or too dangerous for humans, often with a level of precision that exceeds human capabilities or reducing cost as compared to sending a human into a difficult to access environment. A typical teleoperated robotic system consists of a robot and an interface from which a human operator can control movement or other functions of the robot, usually through a set of joysticks or using a computer mouse to click buttons in the interface [25, 49]. Commonly, the operator interface also provides visual access to a live video stream from a camera mounted on the robot. The distance

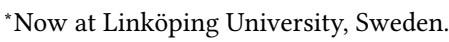

Authors' addresses: Hannah R. M. Pelikan, University of Twente, Enschede, The Netherlands, h.r.m.pelikan@student. utwente.nl; Amy Cheatle, Cornell University, Ithaca, NY, USA, ac2288@cornell.edu; Malte F. Jung, Cornell University, Ithaca, NY, USA, mjung@cornell.edu; Steven J. Jackson, Cornell University, Ithaca, NY, USA, sjj54@cornell.edu.

Permission to make digital or hard copies of all or part of this work for personal or classroom use is granted without fee provided that copies are not made or distributed for profit or commercial advantage and that copies bear this notice and the full citation on the first page. Copyrights for components of this work owned by others than the author(s) must be honored Abstracting with credit is permitted. To copy otherwise, or republish, to post on servers or to redistribute to lists, requires prior specific permission and/or a fee. Request permissions from permissions@acm.org.

(C) 2018 Copyright held by the owner/author(s). Publication rights licensed to the Association for Computing Machinery. 2573-0142/2018/11-ART138 \$15.00

https://doi.org/10.1145/3274407

Proceedings of the ACM on Human-Computer Interaction, Vol. 2, No. CSCW, Article 138. Publication date: November 2018. 
between the robot and the operator who controls it varies greatly, from a few meters to millions of miles. For example, a surgeon may just sit a few meters away when performing complex minimally invasive surgery with the help of a robotic system. Operators of robots that are used to support disaster response teams [15] or teams in the military tasked with disposing explosive ordnances [14] might operate the robot from a few hundred meters away. Military drone operators may sit half a world away from the sites and targets of military strikes [3]. Robots used in space exploration may be millions of miles away from their human operators [83].

Distance has long been recognized as an important aspect and challenge of technology supported cooperative work. Controlling a robot from a distance brings many challenges well documented in the literature of $\mathrm{CSCW}$ and allied fields, including limitations in perception through the sensors of the robot $[12,15,83]$, a resulting lack of situation awareness [12, 21], and difficulties in building shared mental models with other team members $[12,15,58,83]$. As robot operators often collaborate with workers that are at a distance from their own location [58], they may also encounter challenges common to remote collaboration more generally, such as difficulties of maintaining common ground $[19,38,63]$, limitations in nonverbal communication and establishing joint attention [47] and experience a reduced influence of telepresent team members on a larger collocated team [78]. Distance has also been found to matter when people interact directly with telepresence robots $[76,82,84]$ as people have been found to apply social norms about appropriate distance [29] directly to robots.

Robotic surgery represents a special form of teleoperated-robot supported work. In contrast to other cases in which the robot operator is a specialist that is added to the team, in robotic surgery an existing team member, the surgeon, who is also leader of the team, takes on the role of the operator. A robotic surgical system like the Da Vinci Surgical System (Intuitive Surgical, California, USA) consists of a large robot with several arms to which surgical instruments and an endoscopic camera are attached. The instruments enter the patient's body through small incisions about the size of a fingertip (making robotic surgery minimally invasive) rather than through a long cut, as in open surgery. The operator interface consists of a console through which the surgeon views a 3D video stream from inside the patient, captured by the camera on one of the robot arms. The surgeon controls movement of the instruments that are mounted on the robot arms with joysticks. Figure 1 provides an overview of a typical setup for robotic surgery, in which the surgeon (on the right) is seated in front of the console, unscrubbed and turning his or her back on the patient (left). The robot (center) takes up most of the space around the patient, where usually the team is huddling.

The Da Vinci Surgical System teleoperation robot is the most popular robotic surgical system in current use, with about 877,000 surgeries performed with the system worldwide in 2017 [37]. It is used for all kinds of surgeries, in the specialties of general, gynecologic, urologic, colorectal, cardiac, thoracic as well as head and neck surgery. The premise of this technology is that the effectiveness of surgical procedures can be greatly improved by overcoming a surgeon's physical limitations [62]. Research has demonstrated several advantages over traditional open or laparoscopic ${ }^{1}$ techniques. Robotic surgery holds several advantages over other currently available techniques (see inter alia $[10,42,67])$. It can achieve higher precision due to reduced tremor and motion scaling. Vision is improved as compared to laparoscopic surgery, in which surgeons have to rely on a $2 \mathrm{D}$ video stream, and the possibility of magnification allows access to more visual detail than is available to the bare eye in open surgery. Further, the robot reportedly reduces surgeons' cognitive load and

\footnotetext{
${ }^{1}$ Open surgery: tissue and organs are fully exposed by a large cut, and the surgeon manually manipulates tissues and organs in the cavity. Laparoscopy: instruments are inserted through small incisions ("keyholes"), the cavity is viewed through a 2D camera and instruments are inserted on long sticks that surgeon and assistant hold throughout the surgery. Robotic surgery: instruments are inserted through small incisions but the robot holds them in place, surgeon and resident view the cavity in 3D through large consoles and manipulate instruments through joysticks in these consoles.
} 


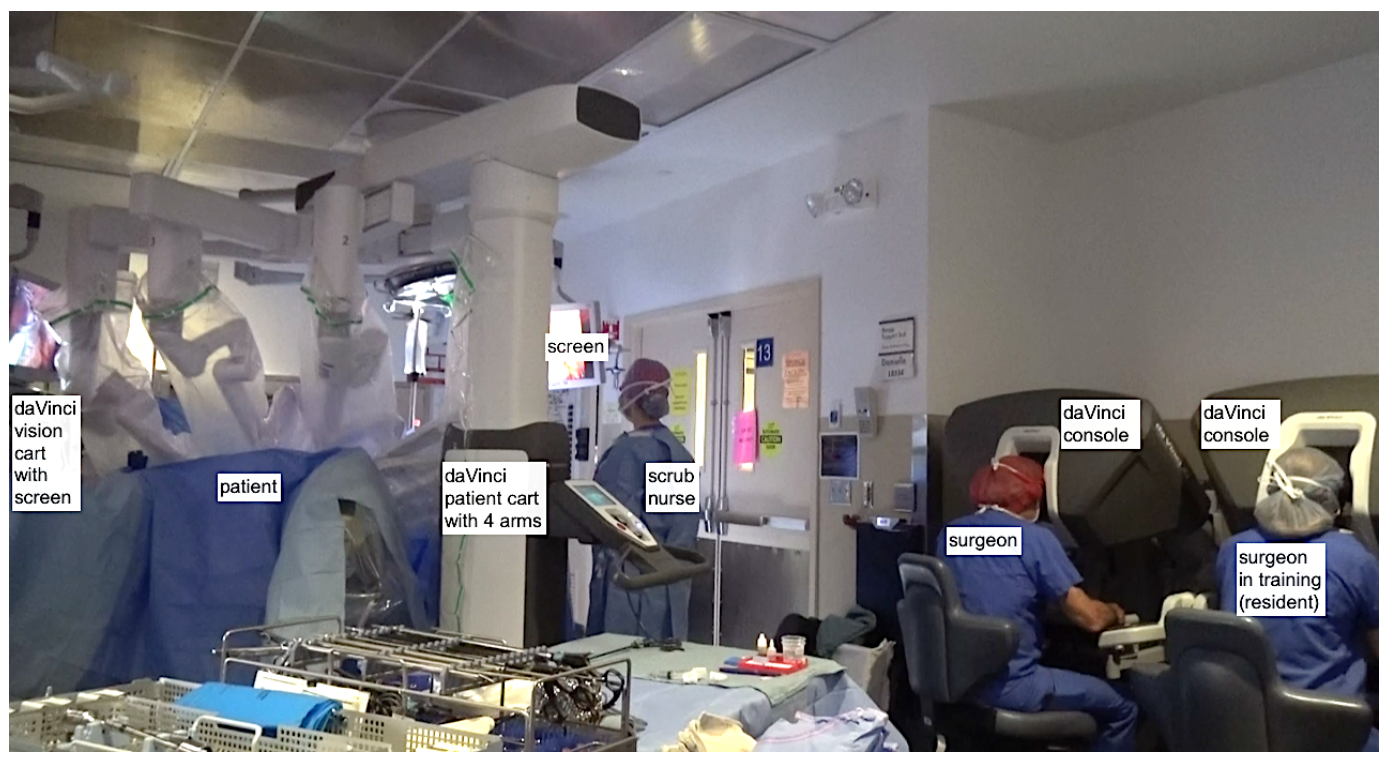

Fig. 1. Typical setup during robotic surgery. Two surgeons are operating from the consoles on the right, which provide them with $3 \mathrm{D}$ vision. They are controlling the patient cart on the left, with its four arms moving inside the patient (under blue drapes). 2D endoscopic video stream from inside the patient is displayed to other team members on several screens distributed within the room (e.g. middle, with nurse watching closely).

physical fatigue, allowing for longer and more efficient scheduling and perhaps more ergonomic working conditions [51]. While some evidence is mounting that these technical advances lead to improved patient outcomes such as reduced recovery time and blood loss [10, 26, 52, 67], there is still debate over whether robotic surgery allows significant improvements for patients over laparoscopic surgery and whether eventual benefits are justified by increased costs $[18,28,66]$.

While a considerable amount of research has focused on understanding how the use of the robot impacts the practice and performance of the individual surgeon-operator, we know much less about its immediate and wider effects on the surgical team as a whole. A small body of research has begun to tackle this question, suggesting that the robot affects communication patterns and leads to increased verbal communication $[20,50,60,72,74,86]$. The robot has also been found to affect teamwork by changing task distribution $[2,85]$. To our understanding, no research has explored specifically how the spatial displacement of the surgeon from the body of the patient and the huddle of the team (as is typical in open surgery) to a more peripheral position at the robot console affects surgical teamwork and experience more generally. Drawing on the well-established insight that "distance matters" in shaping the collaborative practice and experience of teams $[12,15,63]$, and focusing on enacted work practice [65], the following questions emerge:

- How do teleoperated surgical robots reconfigure the spatial arrangement of surgical teams in the operating room?

- How does this spatial reconfiguration affect and remediate the work of broader surgical teams, including in their material, experiential, and affective dimensions?

Drawing on ethnographic observation, interviews and video analysis of surgeries conducted with and without the Da Vinci Surgical System at two hospitals in the USA, our work makes three central contributions. First, we provide a detailed understanding of how introduction of a teleoperation 
surgical robot reconfigures the spatial arrangement of existing teams. Second, while most studies of robotic surgery focus on the impact of the robot on the surgeon and patient, our research focuses on the team and provides evidence that the use of the robot fundamentally changes how surgery is practiced by the entire team. Third, we introduce a framework that helps to understand how tele-robotic technology influences the interplay between space, task-related and interpersonal aspects, by highlighting how changes in physical distance might lead to changes in what we refer to as cognitive and affective distance.

The paper is structured as follows: In section 2, related work is introduced, covering work on (robotic) surgery, teleoperation and distance collaboration, as well as proxemics. The ethnographic method is described in section 3, illustrating data collection in the hospital and providing details about the robot and team members. In section 4 , the relationship between distance and collaboration is analyzed from three perspectives, starting with physical aspects and moving on to task-related cognitive distance and interpersonal affective distance. Finally, findings are discussed along our proposed framework in section 5, pointing out implications for power distribution, team affect and future applications of our framework before the paper concludes by summarizing key points in section 6.

\section{RELATED WORK}

The nature of surgical work is deeply cooperative, as evident for example in the joint act of passing instruments between scrub nurse and surgeon $[8,80]$, coordination between surgeon and team in providing and following instructions [57, 81], dynamic coordination of embodied actions in anesthetic teams based on team members' close mutual monitoring [32,33] and the collaborative sense-making of the patient's body and surgical landmarks in laparoscopic surgery [43-46, 55]. The cooperative character of surgical work is also reflected at a higher level, as seen in collaborative time management for surgical scheduling $[4,6,22]$ or coordination between hospitals that a patient is treated in, starting from diagnosis and preparations, to the actual surgery to after care [27] Surgical work is characterized by a complex and long-established division of labor, and surgical team members rely heavily on each other's skills $[35,88]$. At the same time, there is also a clear hierarchy: authority lies with the surgeon, who is responsible for actions in the operating room $[35,88]$. Hirschauer [35] speaks of a "surgeon-body", pointing to an orientation in which team members are seen as functional extensions of the surgeon's acting body. Personal boundaries are subordinated to the functioning of the team, and it is common for the surgeon to rely on other's bodies, for example when moving assistants' hands into a preferred position or asking nurses to wipe sweat from his or her forehead [35]. A unique characteristic of surgical teamwork is also its close spatial organization in comparison to other types of teamwork [54]. Team members gather around a prone and anesthetized patient, who is positioned at the center of a shared interactional space and stand very close to each other [54], regularly making intimate bodily contact to achieve their tasks [8].

There is mounting evidence that robotic surgery affects a team's surgical practice in a number of ways. First, the introduction of teleoperated surgical robots changes a team's spatial arrangement, separating team members in space [75] and placing the surgeon at a distance from both patient $[64]$ and team $[2,50,73]$. Further, the robot has been found to affect communication patterns [13] leading to increased verbal communication [20, 50, 72, 86]. Nyssen and Blavier [60] found a significant increase of the number of communication acts in robotic surgery as compared to laparoscopic surgery. Reasons for the increase in communication are a lack of situation awareness of the surgeon $[72,73]$ and an increase in information flow, as surgeon and team need to exchange information about the state of the robot $[13,86]$. Communication in the presence of the robot has been described as more difficult, as the robot introduces "an auditory, visual, and physical 
barrier between team members" [75, p. 3]; it may be harder for team members to hear each other over the additional noise from the robot, something even the microphone system built into the robot cannot fully compensate for. In addition, both vision and freedom of movement may be impaired, as the robot takes up a large and usually central space in the operating room (including crucial patient-side space). The robot thereby challenges - and in some cases outright prohibits - certain forms of face-to-face interaction through large parts of robotic surgery [60,75]. Proper communication is crucial as poor communication quality in robotic surgery has been found to lead to worse surgical outcomes [75].

The robot further redistributes tasks and roles on the surgical team, but research seems contradictory on how this affects teamwork. Some suggest that the robot is taking over tasks from the assistant [85] and task load is redistributed and centered on the surgeon $[2,73]$. Others find that the robot changes roles and workflow in the team and team members have to carry out additional tasks when operating with the robot $[20,50]$. Furthermore, the role of the team is enhanced as they have to communicate information that the surgeon lacks $[72,73]$, whereby the surgeon becomes increasingly dependent on the team [50]. Finally, while teaching and learning is happening through active participation in nonrobotic surgery, trainees get a more passive role in robotic surgery: they spend a majority of their time only watching the surgeon operate on the robot, rather than acting themselves [7].

\subsection{Remote Collaboration in Teleoperation and Distributed Teams}

With the surgeon being at a distance from the team during teleoperation phases, this work shares some commonalities with distributed teamwork. In distributed teamwork team members are not collocated in the same workplace but have to collaborate over distance. Distance collaboration has been found to be challenging for a number of reasons [9, 19, 34, 38, 53, 63]. A major challenge lies in maintaining common ground [17], shared understanding that forms the basis for coordinated action. Team members need to ensure that they share relevant information or at least are aware of information that distant team members do not have $[9,19,38,63]$. Common ground includes taskrelated information $[9,34]$ (e.g. knowledge of medical terms for the surgical setting), information on the process of collaboration $[9,38]$ (e.g. timing of certain surgical actions) as well as contextual knowledge (e.g. that it is a colleague's last day at work) $[19,34,63]$. Thus, team members do not only need to share information regarding the task but contextual and interpersonal aspects also matter. The role of the team leader has been stressed as crucial for cultivating common ground in tele-robot supported teamwork. Jones and Hinds [38] describe how a remote leader focuses on the task of building a global understanding from information given by team members at different locations, allowing him or her to coordinate actions of the team members according to their local perspective. In tele-robotic surgery, the team leader's major task is to operate the robot, so he or she may only have limited capacities for cultivating a global understanding of what happens in the room and coordinating team members based on this.

Teleoperation and telepresence technologies pose specific challenges on the robot operator. As they only allow limited sensory access, it is hard for the remote operator to maintain situation awareness [24], which is expressed in difficulties to orient in the remote space [15, 21]. Struggles to maintain situation awareness have also been reported for teleoperated surgical robots $[73,74]$ and surgeons generally face difficulties in orienting and identifying structures inside the body when relying on videostream provided by an endoscopic camera [46]. Maintaining common ground is difficult in many teleoperation settings, as not all members of the team that a robot operator collaborates with can communicate with each other [58]. While it is mainly the surgeon and assistant who are communicating in teleoperation phases of surgery, other team members such 
as the circulator can still contact the surgeon by walking over and initiating face-to-face contact when necessary.

Finally, access to resources has been found as a crucial factor influencing collaboration between collocated team members and team members interacting through a telepresence robot [78]. Team members orient towards a telepresence robot when its operator has information which they do not have, but largely ignore the telepresent team member when they themselves have access to this information. In robotic surgery, the surgeon lacks access to information about what is happening by the patient-side and relies on team members to communicate this information [74].

\subsection{Space and Distance in Teamwork}

While we have seen that large, geographic distances influence teamwork, distance is also relevant for collaboration in teams on a more fine-grained level, as distance between people is meaningful for social interaction. Hall [29] has coined the term "proxemics" for the study of how humans use space and orient to distance. The proximity at which people position themselves is determined by their social relationship. Violations of the acceptable distance may provoke strong reactions and both coming too close and staying farther away than expected may cause breaches [11]. In surgery, interpersonal distance is different from conventional social interaction and team members often have to make intimate bodily contact to achieve their tasks [8]. If changes in distance are noted, it seems likely that the increased distance between surgeon and patient as well as surgeon and team will have social implications.

People do not only position themselves at a meaningful distance, but generally organize in space in particular ways. Participants to an interaction usually arrange themselves in specific spatial patterns with a shared interactional space (such as a circle), so-called "F-formations" [41]. Human spatial arrangement is structured not only by the relation to other people but also by objects (e.g. large tables) that invite certain arrangements while at the same time prohibiting others [77]. Similarly, technology impacts socio-spatial arrangements, as its interactive properties demand certain spatial arrangements, which is referred to as "interaction proxemics" [48, 54, 61]. A small tablet for instance requires people to huddle around it to see what it displays, while a larger screen leaves more freedom in spatial configuration [48]. In the operating room, the interaction proxemics of touch-based displays placed far from the operative site have been found to sometimes be at odds with the desired F-formation with the patient at its center [54].

\subsection{Lessons from the Literature}

Our review of the literature highlighted the highly collaborative nature of surgical work. We have seen that surgical work is characterized by strong interdependence of tasks, hierarchy and working in close proximity. We have reviewed evidence that the robot changes a team's spatial configuration, and interpersonal distance. It introduces a barrier between surgical team members, which has documented effects on communication and distribution of tasks and roles. Reviewing broader work on distance in collaboration, we have seen that distance is relevant in a number of settings, as it may affect situation awareness and common ground, and that telepresent team members may have limited influence on the team. Distance also carries social meaning, as people follow certain practices to position themselves in space with a meaningful distance to each other, and this positioning can be mediated by technology. Despite understanding the importance of distance in teamwork and a robot's impact on a team's spatial configuration and interpersonal distance, we have little understanding about the specific ways in which the use of a surgical robot affects a team's spatial configuration, and what consequences this spatial reconfiguration has for how an entire surgical team performs its work. 


\section{METHOD}

Members of the research team carried out ethnographic fieldwork at two teaching hospitals in the USA: a large medical center (H1) and a medium-size community hospital (H2). We shadowed abdominal surgeries with two surgeons in H1, and gynecologic surgeries with one surgeon in $\mathrm{H} 2$. We chose these surgical specialties due to their historical use of the Da Vinci Surgical System, which was approved for these domains by the US Food and Drug Administration (FDA) more than a decade ago. This enabled us to focus our inquiry on surgical practices that have evolved and standardized over time instead of observing teams in moments of flux and change.

Table 1. Overview of Surgical Procedures Included in the Analysis.

\begin{tabular}{|c|c|c|c|}
\hline Surgical Procedure & Type of Surgery & $\begin{array}{l}\text { Hospital } \\
(\mathrm{H} 1 / \mathrm{H} 2)\end{array}$ & $\begin{array}{l}\text { Videotaped } \\
\text { (yes/no) }\end{array}$ \\
\hline Vulvectomy & open & $\mathrm{H} 2$ & no \\
\hline Partial vulvectomy & open & $\mathrm{H} 2$ & no \\
\hline Partial vulvectomy & open & $\mathrm{H} 2$ & no \\
\hline Vulvectomy + sentinel node dissection & open & $\mathrm{H} 2$ & no \\
\hline Cone biopsy & open & $\mathrm{H} 2$ & yes \\
\hline Vulvectomy & open & $\mathrm{H} 2$ & yes \\
\hline Cone biopsy & open & $\mathrm{H} 2$ & yes \\
\hline Partial vulvectomy & open & $\mathrm{H} 2$ & yes \\
\hline Partial vulvectomy & open & $\mathrm{H} 2$ & yes \\
\hline Partial vulvectomy & open & $\mathrm{H} 2$ & no \\
\hline Thyroid surgery & open & H1 & no \\
\hline Partial lymphectomy & open & H1 & no \\
\hline Splenectomy & laparoscopic & $\mathrm{H} 1$ & no \\
\hline Cholecystectomy & laparoscopic & $\mathrm{H} 1$ & no \\
\hline Apendectomy & laparoscopic & H1 & no \\
\hline Partial gastrectomy & robotic $>$ laparoscopic & H1 & no \\
\hline Adrenalectomy & robotic & $\mathrm{H} 1$ & no \\
\hline Bariatric surgery & robotic & $\mathrm{H} 1$ & no \\
\hline $\mathrm{TLH} / \mathrm{BSO}^{a}$ & robotic & $\mathrm{H} 2$ & no \\
\hline $\mathrm{TLH} / \mathrm{BSO}$ & robotic $>$ laparoscopic & $\mathrm{H} 2$ & no \\
\hline Bilateral oophorectomy + node dissection & robotic & $\mathrm{H} 2$ & yes \\
\hline $\mathrm{TLH} / \mathrm{BSO}$ & robotic & $\mathrm{H} 2$ & no \\
\hline Lymph node biopsy sentinel node & robotic & $\mathrm{H} 2$ & yes \\
\hline $\mathrm{TLH} / \mathrm{BSO}$ & robotic & $\mathrm{H} 2$ & yes \\
\hline TLH/BSO & robotic & $\mathrm{H} 2$ & yes \\
\hline Oophorectomy & robotic & $\mathrm{H} 2$ & no \\
\hline TLH/BSO & robotic & $\mathrm{H} 2$ & yes \\
\hline TLH/BSO & robotic & $\mathrm{H} 2$ & no \\
\hline TLH/BSO & robotic & $\mathrm{H} 2$ & yes \\
\hline TLH/BSO & robotic & $\mathrm{H} 2$ & no \\
\hline TLH/BSO & robotic & $\mathrm{H} 2$ & yes \\
\hline
\end{tabular}

${ }^{a}$ Total Laparoscopic Hysterectomy with Bilateral Salpingo Oophorectomy 
Over the course of 14 months we observed a total of 31 surgeries (16 robotic, 15 nonrobotic), 12 of which were videotaped ( 7 robotic, 5 open surgeries). The unrecorded surgeries included 9 robotic, 7 open and 3 laparoscopic cases (see Table 1 for details). In two cases the team switched from robotic to laparoscopic surgery towards the end of the procedure; we counted them as robotic cases here. We observed various types of surgery, all of them centered on the patients' torso. Gynecologic surgeries included hysterectomies (partial or full removal of uterus), oophorectomies (partial or full removal of one or both ovaries), vulvectomies (partial or full removal of vulva) and cone biopsies (removal of a cone-shaped piece of cervical tissue). While the former two are usually performed with the robot, the latter two are typical non-robotic procedures. Surgeries shadowed in H1 included an apendectomy (appendix removal), a cholecystectomy (gallbladder removal), a splenectomy (spleen removal), an adrenalectomy (removal of the adrenal gland), a thyroid surgery, gastric surgeries (partial gastrectomy, partial lymphectomy) and a bariatric (weight loss) surgery. The decision on whether the procedure would be robotic, laparoscopic or open depended on many factors, and varied between hospitals. Major factors are FDA approval for the specific type of surgery as well as the patient's individual body type (e.g. with morbidly obese patients laparoscopy is not possible, meaning surgeons must turn towards robotic procedures as the only viable minimally-invasive option). In H1, the robot was shared with other departments, and scheduling conflicts meant the robot was not always available to both. Cost can also play an important role in hospital decisionmaking processes, as robotic surgeries are significantly more expensive than laparoscopic or open surgeries. However, surgeons at our fieldsites did not report cost to be a contributing factor in their planning for robotic cases. We interviewed different stakeholders to represent different perspectives (see Table 2), such as surgical staff (6 surgeons at hospitals in the US and Europe, 3 residents, 1 intern, 1 student, 4 first assistants, anesthetist, 7 scrub nurses, 6 circulator nurses, 1 charge nurse), representatives of the robot manufacturer Intuitive Surgical, as well as a human factors expert at the FDA, who was involved in the process of granting approval for the Da Vinci Surgical System (see 3.3). Our general focus was on teamwork between surgical team members with and without the surgical robot. We included all collected data in the analysis.

Table 2. Interviewee Roles and Number of Interviewees in these Roles.

\begin{tabular}{|c|c|c|c|}
\hline \multirow[b]{2}{*}{ Role } & \multicolumn{2}{|c|}{ Interview setting } & \multirow[b]{2}{*}{ Total } \\
\hline & Formal & Informal & \\
\hline Surgeon & 6 & - & 6 \\
\hline Resident & - & 3 & 3 \\
\hline Intern & 1 & - & 1 \\
\hline Student & - & 1 & 1 \\
\hline First assistant & 2 & 2 & 4 \\
\hline Scrub tech nurse & 1 & 6 & 7 \\
\hline Circulator nurse & - & 6 & 6 \\
\hline Charge nurse $^{a}$ & 1 & - & 1 \\
\hline FDA human factors expert & 1 & - & 1 \\
\hline Intuitive Surgical representative & 2 & - & 2 \\
\hline Total & 14 & 18 & 32 \\
\hline
\end{tabular}

Formal interviews were scheduled ahead of time and were conducted via teleconferencing tools or the telephone. Informal interviews were conducted in the field adjusting to in situ events and participants' work practice.

${ }^{a}$ In charge of scheduling and facilitating distribution of resources. 


\subsection{Data Collection}

Two of the authors carried out observations, separately joining surgical teams in the operating room. We stayed with the nurses, usually entering the operating room when preparations for the surgery started and leaving after the room had been cleaned following the last procedure of the day. We collected detailed ethnographic fieldnotes during visits at both hospitals, while video data was recorded in $\mathrm{H} 2$ only as staffing in the robotic team at $\mathrm{H} 2$ was generally more consistent and stable when compared to H1. This enabled us to build trust over a longer period of time, ensuring consent to record could be obtained. We found it helpful to only gradually bring camera equipment into the setting, after building trustful relationships with the participants, as suggested by [30] For videotaping, we used two camcorders and two GoPro cameras and an additional high-end microphone placed at different angles in the room in order to capture activities of the surgical team (see Figure 2). We obtained informed consent before switching on recording equipment at all times. As we recorded no patient information, we obtained consent from the patient verbally before anesthesia administration. We informed patients that focus lay on the surgical team and camera equipment would only be switched on after the patient was fully draped and therefore not identifiable. All but one patient agreed to having their procedure videotaped. In total, we collected 68 hours of audiovisual data. Time codes of interesting events were registered in the fieldnotes to facilitate retrieval of those instances from the data. In addition to the observations, we conducted semi-structured interviews, organized around different roles of the surgical team, benefits and challenges of working with the robot, and team affect. Due to the specific working conditions and work schedules, we decided to conduct some of the interviews in situ and more informally, recording them on video and in the fieldnotes (see Table 2 for details). Nurses were often happy to answer questions while preparing the room or waiting for the patient to arrive, but reluctant to schedule a formal interview outside their working hours. The interviews labeled as formal were conducted via teleconferencing tools or the telephone, each taking about an hour. All interviews were transcribed and used to guide subsequent observations and interviews. All data is stored on a password protected cloud drive, which only members of the research team have access to.

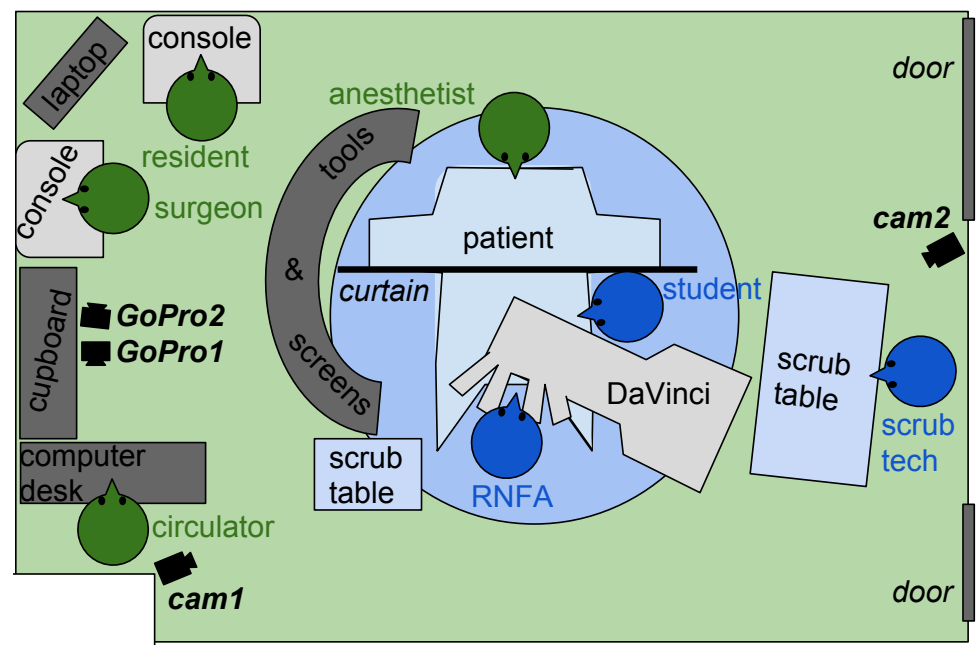

Fig. 2. Layout of the room with camera positions. Sterile field in blue (circle), with scrub tech, student and first assistant (RNFA) in sterile gowns. Circulator, surgeon, resident (surgeon in training) and anesthetist are working outside the sterile area. 

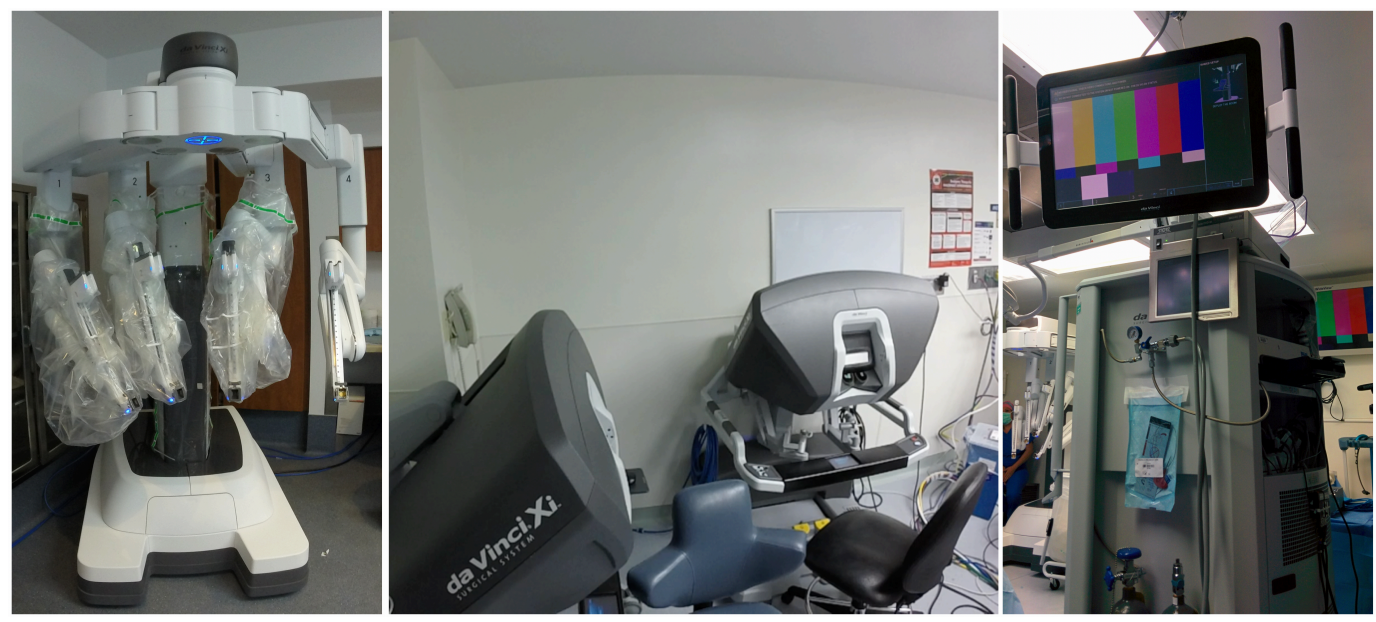

Fig. 3. The Da Vinci Xi Surgical System. Left: The robot patient cart with four arms, three of which are already covered in sterile drapes. Middle: The consoles through which the robot arms are controlled. Right: Vision cart doing the computations and displaying high-quality video from inside the patient.

\subsection{Data Analysis}

Data was analyzed using a grounded theory approach $[16,23,79]$, a form of data-driven, openended and inductive analysis characterized (as deployed here) by an orientation towards ordinary and mundane practice rather than extraordinary events. During an initial phase of open coding, fieldnotes, interviews, and video data were carefully reviewed and sorted to form an initial overview of project materials. In a second step, key themes in the data were identified and discussed by members of the research team; one of these, "new forms of distance in robotic surgery", was selected as the main theme for the current paper. Several subthemes (physical, cognitive, affective, and hierarchical distance) were then identified based on salient themes in the data. A period of focused coding was then undertaken, during which all data was systematically re-coded and fieldnotes, interview data and video clips related to the selected subthemes were identified and compiled. These data slices were then compared and contrasted with each other, identifying similarities, variations and larger and recurring patterns in the surgical teams observed.

\subsection{The Surgical Robot}

The teleoperation robot used in the majority of surgeries was a Da Vinci Xi Surgical System. The system consists of three components (see Figure 3): The patient cart (left) is about the size of a large refrigerator and has four extendable arms that are operating inside the patient. The surgeons control movement of the arms on the patient cart through the consoles (middle), which provide $3 \mathrm{D}$ video feed from inside the patient. The vision cart (right) contains all computing equipment, a microphone system as well as a screen that displays the video feed in $2 \mathrm{D}$ for the team. A small number of surgeries was carried out with the previous model of the robot, the Si system, which is similar in size and shape but only has three arms.

\subsection{Surgical Team Members}

Surgical teams consist of several team members with different roles and skills. The surgeon acts as head of the team and carries out major parts of the surgery. Many surgeries also include a first assistant to help the surgeon, which is often a specifically trained nurse (Registered Nurse 
First Assistant, RNFA). An anesthetist narcotizes the patient and monitors vital life functions during the surgery. A scrub tech works in the sterile field and hands over instruments to the surgeon, anticipating what tools will be needed next $[8,80]$. Further, a circulator is needed to fetch instruments from the non-sterile areas and to count sponges and tools throughout the surgery to ensure nothing is left in the patient. The circulator also documents the surgery in the computer, noting for instance administered medicine. Surgeries in teaching hospitals like the ones that we conducted our research in may also include a range of residents, interns, and students, observing and occasionally participating in elements of the surgical procedure. Thus, while a surgical team consists of at least 5 members, in teaching hospitals it is often 8-10 people. Complicated surgeries are often staffed in addition with several scrub techs and circulators.

\section{ANALYSIS}

\subsection{Reconfiguration of Physical Distance}

Comparing robotic with non-robotic surgery, we observed a reconfiguration of space in general and distance between team members in particular. We distinguish three ways in which the robot spatially reconfigures the team by (1) displacing the surgeon from the team, (2) scattering team members by taking their space around the patient and (3) introducing a visual barrier between team members. The following two vignettes, brief fieldnote-based descriptions, set the scene and illustrate how the robot changes distance as compared to non-robotic surgery. Vignette 1 depicts how team members are in close physical contact during non-robotic surgery. During the teleoperation phase of robotic surgery distance changes between all team members, which is described in Vignette 2. In the following vignette, the surgical team is performing a partial vulvectomy.

\section{Vignette 1. Physical Distance in Open Surgery.}

The surgeon and his team are gathered around the patient. The surgery has just started and the surgeon and his intern, a first-year surgeon-in-training, are discussing the procedure in a low voice. The two of them are squeezing into the narrow space between the patient's legs, their heads are almost touching as they bend forward to take a closer look. The surgeon is inspecting the patient with his hand. Behind the intern, a student has positioned himself to closely watch the procedure, looking over their shoulders. The scrub tech is standing by the patient's left leg and watching the surgical work, getting tools ready on her scrub table as she anticipates that they will be needed soon. The surgeon requests the electrocautery device to be connected and starts teaching his intern how to set the cuts for a vulvectomy. He lets her hold the pen-like device and then grabs her hand and directs it, guiding not only the direction of the cuts but also teaching her the right pressure. They work focused and quietly, the surgeon giving instructions or checking back with the anesthetist in a low voice every once in a while. The circulator watches the procedure standing close to her desk, ready to make calls or fetch tools that are not on the scrub table. The space around the patient is crowded, the entire team is huddled together, less than an arm's length from each other.

The vignette illustrates the closeness of surgical team members and patient during open surgery. A similar description holds for laparoscopic surgery. The team gathers around the patient in an F-formation [41,54], arranged in a circular pattern around the patient, who is at the center of their shared interactional space. The team members are huddling in the limited space and surgeon and intern are so close to each other that their bodies are touching. The surgeon directs the learners' hands to teach them how movements and tissue should "feel". The other team members stand close by, peeking over their shoulders to anticipate subsequent actions. Team members and patient are 
literally "in touch" with each other. As team members can see (and feel) each other, a lot of the communication is happening nonverbally, as for example during the constant instrument handovers between surgeon and scrub tech. The circulator can easily check back with the team by walking over to the patient side.

Distance is more variable in robotic surgery. During preparation of the surgical site for the robot, the team is grouped around the patient in a similar way as during non-robotic surgery. Docking the robot is a crucial moment in which all team members get involved: the circulator is usually moving the robot closer to the patient, struggling not to get too close and hit the patient. The other team members are guiding this movement. Once this is accomplished and the arms are set into the correct positions, the teleoperation phase begins. The team spreads out and distance between team members increases dramatically. Vignette 2a illustrates how the surgeon is displaced from the team when the robot comes into action.

\section{Vignette 2a. Physical Distance in Robotic Surgery: Surgeon Perspective.}

As the robot is docked into place, surgeon and resident remove their sterile coats and gloves and move to the consoles at the far end of the room, which are hidden behind anesthesia carts and screens. Turning their backs on the patient, they take a seat and bend their heads down, pressing their face into a viewfinder, which looks much like giant virtual reality goggles. Someone has turned the lights off. The surgeon instructs his resident, who is sitting about two arms distance away from him at the other console, immersed into three-dimensional live image from the patient's abdomen. The surgeon's voice is picked up and amplified loudly into the room over the speakers. He says "go ahead", which is followed by the deep humming sound of the cauterizing tool.

As the vignette illustrates, surgeon and resident are physically removed from patient and the rest of the team to a corner of the room, turning their back on the patient. In some operating rooms consoles were also physically separated from the rest of the room by an arrangement of screens and other machines. Once surgeons lean into the consoles, they see the patient's body from the inside, but at the same time, visually dislocate themselves from their surroundings in the OR. Unless the surgeon leaves the console, establishing eye contact with others becomes impossible. The robot thus does not only physically dislocate the surgeon to a corner of the room but also prohibits face-to-face interaction with the team. Surgeon and resident are also at a larger distance from each other as compared to open surgery, where they are often standing shoulder to shoulder and the surgeon can direct students' and residents' hands when teaching.

As the surgeon is removed from the patient-side in robotic surgery, an additional team member, a first assistant, is needed to support the surgeon from the patient-side, controlling the suction machine and changing the robot instruments. The first assistant is also largely mediating interaction between nurses and surgeon in robotic surgery. The following vignette illustrates how by its large size, the robot distributes team members in space and introduces a visual barrier between them.

\section{Vignette 2b. Physical Distance in Robotic Surgery: Team Perspective.}

Between the patient's legs, the first assistant is pulling back the wide transparent plastic drapes around the robot arms, trying to get a better view of the student, who is standing by the patient's chest doing suction. The patient is positioned in an extreme angle, her head only a few inches from the floor and her legs spread high up in the air. Her entire body is covered in light blue drapes, leaving only a small square area of skin visible. This is where the four robot arms are inserted, moving up and down. Live video-stream from inside the patient is projected onto three large LCD screens spread throughout the room, making the surgeons' actions available to everyone in the 
operating room. The anesthetist is hidden behind a light blue curtain, kneeling down to give medication. Behind the robot base, the scrub tech is sorting the instruments on her table, slightly moving from one foot to the other. Two circulators are talking in the corner by the circulator desk, showing each other pictures on their phones. The team is scattered throughout the operating room, out of view from each other.

As illustrated in this vignette and Figure 2, team members scatter throughout the room in the teleoperation phase, leaving the original F-formation with the patient at the center. As the robot takes up a majority of the space by the patient, team members have to arrange themselves with respect to the robot. This becomes most evident for the role of the scrub tech, one of the most important actors in open and laparoscopic surgery, whose usual place close to the patient is literally taken by the robot. During non-robotic surgeries, scrub techs stay about an arm's length from the patient anticipating subsequent actions and skillfully handing instruments to the surgeon, often communicating nonverbally (see e.g. $[8,80]$ ). When the robot is switched on and driven to the patient, the scrub tech has to move the scrub table to give way to the robot who is taking up this space. The scrub tech moves further away from the patient, positioning the scrub table behind the robot base. Thereby the scrub tech is not only positioned further from the patient but also from the rest of the team members who work in the sterile area. Having to plan all movements around the robot, the circulator is more restricted in his or her movement during robotic surgery. Even before the robot is docked, it is taking up a considerable amount of space. In small operating rooms, we have seen circulators crawl through under the robot arms, trying to avoid touching the sterile robot arms that were blocking the way. When interacting and sharing information with the team, the circulator can no longer address them all at once by moving to the sterile area around the patient, but he or she has to move to different locations, depending on the addressee. In such cases, her mobility is increased as she is trying to obtain the right configuration of information and resources to complete her tasks [5].

The robot does not only distribute team members in space, but also introduces a visual (and auditory) barrier. As the robot is very large and the arms are wrapped in large plastic drapes, the robot occludes sight and prohibits face-to-face interaction during large parts of the surgery. When the first assistant is positioned between the patient's legs, he or she cannot see what is happening around head and torso of the patient. This is especially problematic when students or interns are tasked with changing robot arms and doing suction, as neither the surgeon, nor his representative by the patient, the first assistant, can see what the learners are doing and largely have to instruct them verbally. When the first assistant is positioned by the patient's chest, he or she largely loses visual access to the rest of the room. Similarly, the anesthetist is visually cut off from the team by the robot. In many open cases, a drape shields the operative area from the anesthetist. In robotic surgery, a curtain is put up, separating the anesthetist from the sterile robot area. He or she is thereby cut off from most of the action in the room and the team members only learn about anesthesia struggles when he or she verbalizes them. In many cases, the robot also blocks sight between first assistant and scrub tech as well as scrub tech and circulator. While they could anticipate subsequent actions by watching the work of the others or communicate nonverbally, they now explicitly have to call each other in a loud voice when they need help. While the robot takes up a lot of space and blocks view inside the OR, it allows to see what is happening inside patient, as the surgeon's actions inside the patient are projected on large screens spread throughout the operating room. Even if it is only in 2D, it increases the team's visual closeness to the patient, allowing them to see much more detail than during open surgery. This also affects the nurses' practice of predicting next actions: In open surgery scrub tech and circulator largely rely on the 
surgeon's nonverbal conduct to predict which tools will be needed soon. In robotic surgery, they orient to surgical actions displayed on the screens as relevant for predicting next tasks.

However, the robot does not only increase physical distance, but rather makes it more variable, introducing a fluid back and forth between large distance and close proximity. Team members start huddled around the patient, then spread out during the teleoperation phase. They come together again to inspect the specimen (the organs and tissue they cut), and then spread once more to close inner wounds. They finish huddled around the patient, closing the incisions once the robot is removed. Open and laparoscopic surgeries, in contrast, are more static, with the whole team centered around the patient and only the circulator moving and fetching instruments every now and then.

\subsection{Reconfiguration of Cognitive Distance}

As the robot alters physical distance, it also reconfigures cognitive aspects of surgical team work, such as sharing information and building common ground related to the task. Spatial rearrangement in robotic surgery results in a reorganization of information access. As team members do not have access to the same information at the same time any more, additional communication work is needed to maintain common ground. We use the term cognitive distance here to refer to distance that emerges along cognitive dimensions such as the conceptual distance that exists between different understandings of the same situation that two people hold. First, we will illustrate how the surgeon has to rely on the team to provide information about what is going on by the patient-side, as the surgeon's situation awareness is impaired. Second, we demonstrate how team members and surgeon negotiate common ground about events that only the surgeon is aware of. Finally, we show how the robot affects attention of the team.

4.2.1 Impaired Situation Awareness on Surgeon's Side. Our video data and fieldnotes hold various examples of the surgeon checking back with his first assistant, in order to make sure understanding about a patient is shared. For instance, we observed a surgeon instructing his resident to be careful about not scratching the bladder while maneuvering towards the uterus, when he suddenly stopped his talk and initiated the following exchange:

\section{SurgeonD: $\quad$ You're pushin', right? \\ First assistant: Pushin', Sir}

With a brief sentence, the surgeon asks for confirmation from his assistant on whether he is pushing. He is referring to an instrument that marks the uterus, which when pushed, makes it easier for surgeon and resident to identify the organ. The first assistant responds in an equally short phrase, confirming that he is pushing and ending his statement with "Sir". As the surgeon's situation awareness about what is happening at the patient side is impaired, increasing cognitive distance, he or she needs to rely on information from the team to maintain common ground. We have observed various teams successfully communicate using this form of brief exchanges, a practice similar to so-called "readback" [74] in aviation communication, in which all information is repeated, keeping the length of utterances at a minimum and completing with a recognizable statement ("Sir" is used equivalently to "over" in this setting). The surgeon does not use a specific addressing term, but signals to the assistant that this is directed at him by increasing volume of these phrases.

4.2.2 Impaired Situation Awareness on Nurses' Side. Often the surgical staff does not have the same informational resources as the surgeon, even though the surgeon's movements of the instruments are made available on screens distributed throughout the room. As the surgeon views the video stream in 3D and is moving the tools, he or she has additional information, of which the team is not aware. Therefore, if the surgeon is encountering trouble, he or she needs to verbalize 
it and bring the issue to the team's attention. This is in stark contrast to open surgery, where all team members share situation awareness and trouble is dealt with mostly nonverbally, without interrupting the flow of the interaction [31]. In the following example, the surgeon needs help but first has to "convince" his team members of the cognitive distance between them, explaining that something is wrong with the endoscope, hindering his sight. He asks his assistant by the patient-side about the camera, but it turns out that he has not noticed any trouble yet. With the help of the circulator, who can touch the non-sterile vision cart, the video feed on the screens is changed, making the trouble available for the rest of the team, who only then have the informational resources to jump to help.

Vignette 3. Negotiating Common Ground.

The surgeon complains about the endoscope. At first glance, the image on the screens appears normal. The surgeon gives an explanation, since the rest of the team seems to share this impression. He says that the camera image on his right eye is totally blurry. They switch the camera feed on the screens and suddenly everything is heavily blurred, making it hard to recognize blood vessels and smaller structures. The first assistant grabs a towel from the scrub table and gets ready to clean the endoscope.

\begin{tabular}{|c|c|}
\hline SurgeonD: & What's wrong with this camera today? \\
\hline First assistant: & Why, what's wrong with the camera, Sir? \\
\hline SurgeonD: & $\begin{array}{l}\text { I can't get it clean it either } \\
\text { (cauterizing for } 3 \text { seconds) }\end{array}$ \\
\hline First assistant: & Which eye is it? \\
\hline SurgeonD: & $\begin{array}{l}\text { The right } \\
\text { (4 seconds silence) }\end{array}$ \\
\hline First assistant: & Lucy $^{a}$ \\
\hline Circulator: & What? \\
\hline First assistant: & $\begin{array}{l}\text { Can you hit the right eye button } \\
\text { so we can see exactly what it is like that- }\end{array}$ \\
\hline $\begin{array}{l}\text { Surgeon: } \\
\text { Circulator: }\end{array}$ & $\begin{array}{l}\text { Go to the right, it's totally blurry } \\
\text { (presses button for right eye view) } \\
\text { (looking at screen) Oh }\end{array}$ \\
\hline First assistant: & (looking at screen) Oh alright \\
\hline
\end{tabular}

The vignette and video transcript above show how the team is joining efforts to address cognitive distance between them. As the team does not share situation awareness, they need to increase efforts to negotiate common ground of what is happening with the camera. The above exchange took roughly 30 seconds. This may not seem much, but in surgery, every second counts and such extra efforts matter, especially if similar situations occur repeatedly. Further, we would like to point out that this is a highly-coordinated team that has worked together for several years. An unexperienced group might have taken much longer to recognize the lack of shared situation awareness.

4.2.3 Difficulties in Maintaining Awareness. In the above paragraphs, we have shown how team members struggle to maintain shared situation awareness and negotiate common ground. We want to have a closer look at challenges team members may face with maintaining awareness of the ongoing activities. The work during robotic surgery is focused on the surgeon, while the rest of the team is left with little tasks, mainly waiting for the surgeon. This results in reduced attention 
and further increases cognitive distance. Several of the surgeons we interviewed described that their team is losing focus on the task:

SurgeonD: [...] There can be extended periods where you don't need anybody else to do anything for you. When that happens they [the team] lose their focus, they start talking, or doing whatever, and you're off in your own little world, and you might need something. Hey, anybody hear me?

Interviewer: You guys?

SurgeonD: You guys pay attention I need help, because they're not going to maintain that focus if it's not needed. It's different than the open surgeries, open you have somebody focused on helping, or doing what they're doing the entire case, this is unfortunately not that way. [...] there's a full-time interaction with your assistants in open cases, so you're both doing the case really. That's not the case with the robot, with the robot you're sitting there by yourself.

Surgeons pointed out that the team does not pay attention in the same way as in open surgery, and they may need to call them several times when they need their help. For different members of the team, this loss of focus may appear in different ways. We found the circulator to often spend time interacting with the computer or their smartphone. We also often saw circulators chat with other team members. Scrub techs in contrast are sterile and cannot touch nonsterile things such as their smartphone. While the major task and skill of the scrub tech is to anticipate which tools are needed and to pass them over at the right time, there is less need for this during robotic surgery. The robot takes over large parts of the work and we found scrub techs to spend most of the time waiting for the robot to be undocked again. Even the first assistant, who is most involved in the surgery because of their regular interactions with the surgeon, spent long periods chatting with the circulator or scrub tech during extended periods of inactivity. While such down times are a normal part of surgical routine, and as surgeons pointed out, are not a problem per se, extended periods of passivity and distraction may become problems when they further impair situation awareness and the team misses important information or fails to react quickly or anticipate when help may be needed. Such problems may mirror those in other human-machine collaborations where automation forces extended periods of passivity: for example, autopilot systems in trains [87] or in self-driving cars [56]. While full focus is not required during large parts of the task, team members need to stay aware of what is happening around them so that they are ready when their actions are needed.

When problems with situation awareness persist and become too large, the fluid form of distance allows the surgeon to get up from the console and move back to the patient, thereby decreasing cognitive distance. This comes at a certain cost, taking time and requiring an additional pair of sterile gloves and a sterile gown that need to be unpacked and put on. Nevertheless, when the team faces trouble and maintaining shared situation awareness verbally becomes too difficult, the surgeon can move over to the patient side any time. Thus the team can move from remote teleoperation to close collaboration in a matter of seconds.

\subsection{Reconfiguration of Affective Distance}

The robot also reconfigures affective aspects of work, such as the team's constant monitoring of each other's wellbeing. As team members heavily rely on each other's skills, losing one team member can impair the entire surgery. Therefore, it is important to ensure that everyone is feeling fine and that team member's emotions and bodily needs (e.g. food, water, warmth) do not affect the surgical outcome. Increased distance between team members in robotic surgery impairs this monitoring. We use the term affective distance here to refer to distance that emerges along affective dimensions such 
as the feeling of emotional disconnectedness that people experience when interacting remotely. First, we will illustrate how team members monitor each other's state in moments where the robot is not present, allowing the team to distribute workload and to maintain positive affect in the operating room. Second, we demonstrate how increased distance between team members in robotic surgery impairs these mechanisms and present an emergent practice developed for dealing with this, namely, the team huddles right before the robot is coming into action, compensating for the distance that they are about to face.

Surgical teams are constantly monitoring the wellbeing of each other, a phenomenon that we observed in different teams and throughout both preparation and the course of surgery. The following vignette illustrates how team members react to each other's affective state and offer help when needed. In this scene, the scrub tech is hurrying to prepare sterile instruments on his table before the surgery when he accidentally drops one of them.

Vignette 4. Displaying sensitivity towards team members' affective state.

Meanwhile, the tech nurse is busy at the tool table, working quickly to prepare with a concentrated look on his face. He drops something and swears. A co-worker comes over and touches him gently on the back. "You seem stressed. Are you?" "Yes", he replies. She offers, "Let me help you set these out" and starts working alongside him to accomplish the task. As the patient and surgeon both are scheduled to arrive soon, he is under pressure.

The fieldnote excerpt illustrates how team members are sensitive to each other's affective state. The scrub tech is setting up the instruments on the sterile table, hurrying to get everything ready before surgeon and patient come in. Another nurse notices that he is stressed and asks him about it. When he confirms, she starts helping him with his task to accomplish it faster. This is only one of many examples, showing how team members communicate their affective state. We found team members to be very open about their affective state, allowing their colleagues to react quickly. Monitoring of affective state does not only happen between nurses, the entire team is involved in this process. Reflecting the surgical hierarchy, nurses have pointed out to us that "the surgeon's emotion sets the whole tone for the room" and a circulating nurse explained that they put efforts into making the surgeon feel safe, explaining that "if the surgeon gets stressed, the team gets stressed."

In robotic surgery, constant monitoring for each other's wellbeing is impaired, as the impaired visual access often results in a lack of awareness of others' emotions. One of the surgeons we interviewed illustrated this, stressing that he is unaware of what his interns are experiencing while he is operating through the robot console.

Interviewer: Do you think there are types of communication that you miss that aren't translated into language or verbal communication? Like cues, I don't know. Do you think-

SurgeonF: Yeah, I do understand what you're saying. I don't think you miss that much that sort of jeopardizes the patient, but if someone had an angry face, or rolls their eyes, I don't see that. Normally, you pick up on that, and respond to that. But if an intern for example feels sick, or feels unhappy with what she's doing, or he's doing I can't see that, they need to speak up.

As the robot obstructs face-to-face interaction, team members are likely to overlook nonverbal cues such as an angry face or an eye roll. Thereby, affective distance is increased. Emotions thus have to be verbalized in order to be noted by the team. It is not only the surgeon who does not 


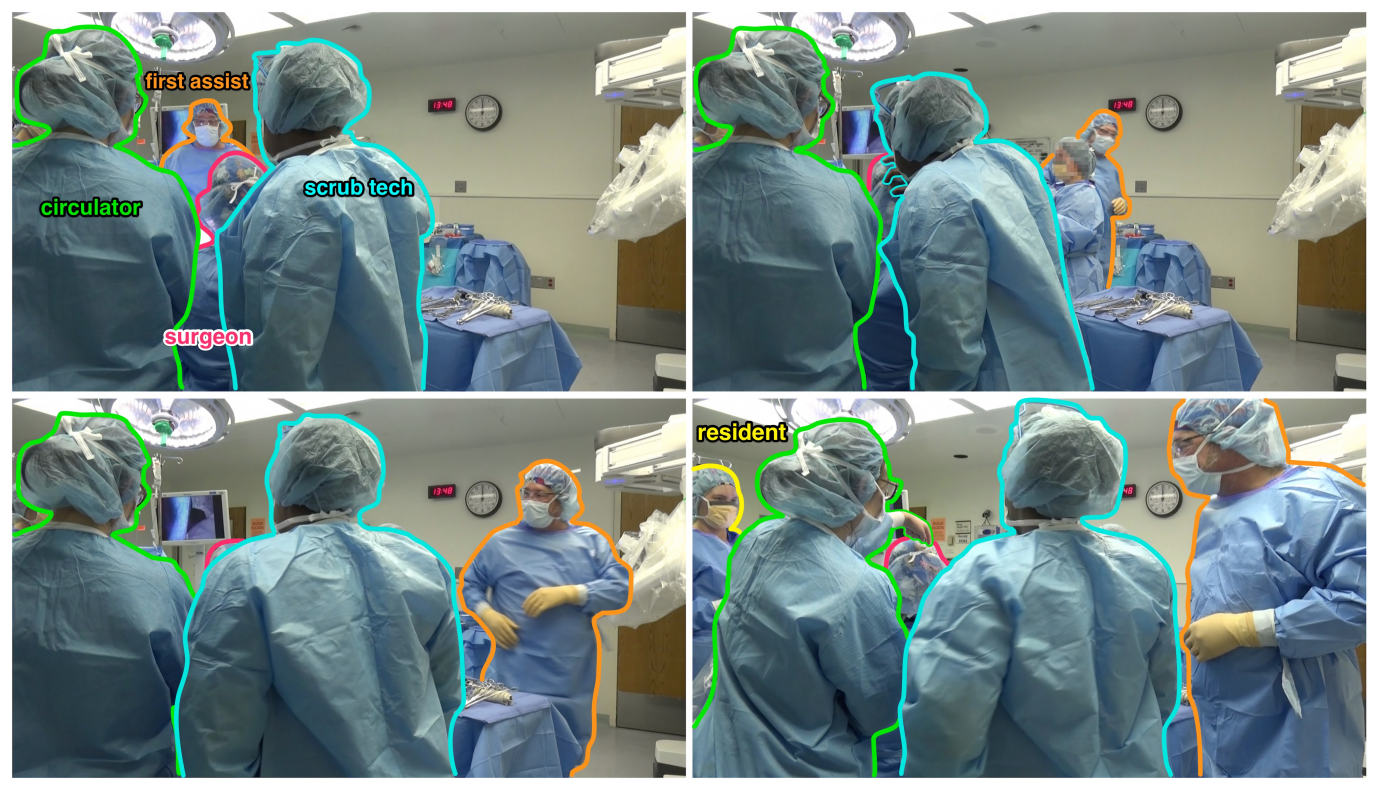

Fig. 4. Team members huddling together before the teleoperation phase. Upper left: Circulator and scrub tech take a look at the surgeon's Halloween-themed cotton cap. Upper right: Scrub tech is pointing out that there is a SpongeBob, first assistant starts moving. Lower left: First assistant walks around patient to take a look. Lower right: Circulator, scrub tech and first assistant are huddling around the surgeon, taking a look at the prints on his cap. The resident (far left) is watching them.

"feel" what is going on with the team, but the team also lacks information about how the surgeon is feeling. One surgeon said that he was "feeling lonely" when he was operating by the console, away from his team.

We noticed several teams huddle before the teleoperation phase as well as surgeons joining their team at the end of a surgery, when assistant, resident and students are closing the incisions. The surgeon is not needed during this task, but seems to enjoy joking with the team for a few minutes before leaving the room. We observed an interesting situation illustrating how the team might be trying to make up for the distance during the teleoperation phase by huddling together before the robot comes into action. In H2, some of the surgical staff was wearing cotton caps under their hair nets. Usually, the surgeon would not wear such a cotton cap, but shortly before Halloween, he came into the operating room wearing one. During preparations of the patient, one of the nurses pointed out that he was wearing a special cap and the others came closer. Figure 4 shows how the nurses gather behind the surgeon, who is seated while vaginally inserting an instrument that will later support the surgery. They huddle together behind the surgeon and carefully touch his head. The first assistant also joins them, and the two nurses point different figures on the cap out to him - SpongeBob, Patrick and a pumpkin. The surgeon lets them touch and gesture behind his head, joining the joking conversation about his cap. The team is in a happy mood and continues to prepare the patient after this brief moment of huddling together just before the robot will separate them.

As the struggles with cognitive and affective distance are brought about by the reconfiguration of physical distance in the operating room, it seems that returning to the huddle of the team helps in addressing these challenges. When cognitive distance cannot be overcome by verbal 
communication, surgeons get up from the console and walk over to to patient and team. Similarly, a strategy to address affective distance seems to be to increase the huddle before and sometimes after teleoperation phases in robotic surgery.

\section{DISCUSSION}

We have shown how the robot reconfigures spatial arrangement of the surgical team in three ways, by (1) placing the surgeon at a distance from the team, (2) scattering team members by taking their space around the patient and (3) introducing a visual barrier between team members. This physical rearrangement changes the way team members collaborate, increasing both cognitive and affective distance. We have shown practices that team members in robotic surgery employ to maintain common ground and when struggling with shared situation awareness, especially as their tasks are increasingly decoupled. We have also shown how affective awareness and sensitivity may be undermined, and the work that surgical teams perform to correct or make up for that lack: for example, by increased interpersonal interactions before and sometimes after the robot comes into action, trying to cope with the reduced affective awareness among the team during teleoperation phases.

We have developed a framework that helps to understand how tele-robotic technology mediates practice by looking at the interplay between space, cognitive and affective aspects. This framework has helped us to understand robotic surgery as a hybrid form of collaboration, which shares characteristics of both distributed and collocated teamwork. We will first discuss this finding and then show how our framework has made this finding possible.

\subsection{A Hybrid Form of Distributed and Collocated Teamwork}

Interestingly, our findings on situation awareness and common ground suggest that work with a teleoperated surgical robot, while being collocated, exhibits phenomena known to be characteristic of distributed teamwork. Challenges with situation awareness to the degree we observed here have rarely been reported for collocated work before. Increased efforts for establishing and maintaining situation awareness have been described for collocated settings that require team members to constantly coordinate with the actions of other team members, while engaging in their own distinct task, such as in news rooms, police operation rooms and traffic control centers [31]. Practices for maintaining awareness in surgical settings in which team members are gathered around the patient, such as microscopic surgery [1] and cases in which surgeon and anesthetist need to work in the same space, on the patient's throat [31] have also been described. However, in these collocated settings, team members largely rely on nonverbal cues to bring certain aspects to the attention of other team members, without interrupting the flow of other ongoing activities [1,31]. To our understanding, previous research on situation awareness in collocated settings has not addressed settings in which the use of nonverbal cues is largely prohibited and in which maintenance of situation awareness therefore has to be negotiated verbally. As team members have to explicitly ask for and share information verbally, the challenges with situation awareness described here for robotic surgery are more similar to distributed collaboration settings.

The idea of robotic surgery as a practice that shares characteristics of both collocated and distributed teamwork is also reflected in our findings on the struggles to establish common ground in robotic surgery. Difficulties in maintaining common ground are typical for remote collaboration $[19,38,63]$. Our examples illustrate how both the remote surgeon and the collocated team members lack crucial information to fully make sense of the situation: the surgeon misses what is happening around him or her as he/she is fully immersed into the patient. On the other hand the other team members have better contextual awareness, but they only seemingly share the surgeon's view as sometimes crucial detail is only accessible through the $3 \mathrm{D}$ perspective that only the surgeon 
has access to, as illustrated in 4.2.2. Similar phenomena are known from distributed teamwork: maintaining awareness of the knowledge that the other side does not have is difficult for dispersed teams, leading teams to assume that they share common ground while they in fact do not [19]. To our knowledge, such problems have not been described for building of common ground in collocated settings. In this sense, while being collocated in one room, the teleoperation phase of robotic surgery exhibits challenges for teams that have only been described for distributed teams to this point.

Finally, the hybrid character of both collocated and distributed teamwork becomes evident in the practice of huddling before and after teleoperation phases, as described in section 4.3. Team members use the collocated phases to build affective ground [40], as the possibilities for doing so are limited during distributed teleoperation phases. Research on distributed collaboration has pointed out the importance of regular intensive face-to-face interaction for (among others) relationship building and communication of complex messages, and effective globally distributed teams have been found to rhythmically intersperse their remote collaboration by face-to-face meetings [53]. Our findings suggest that this does not only apply to distance on a global scale but also holds for shorter distances as in the operating room. The team's practice of huddling before long stretches of being distributed in space is in line with practices that successful global teams apply, showing once again the similarities robotic surgical work, while collocated, shares with distributed collaboration, thereby resulting in a hybrid form of distance work. In the following paragraphs we will detail how our framework led us to this insight, by uncovering practices that would be inaccessible to analysis otherwise.

\subsection{Spatial Distance}

We have seen that the use of the robot changes the spatial positioning between surgeon and team members as compared to nonrobotic surgery. While others have reported changes in spatial positioning of team members as compared to nonrobotic surgery [2, 50, 64, 72, 75], and described the robot as a "barrier between team members" [75, p. 3], they have not described changes in distance between surgeon and team members in a systematic way, as we have done within our framework. We studied how the robot reconfigures spatial positioning of team members in different roles and how it remediates individual team members' distance to each other and to the patient. Thereby, we could gain deeper insights into how technology in the form of a teleoperation robot remediates spatial aspects and distance in particular. Studying distance changes in a systematic way led us to the understanding that given the close and often intimate working conditions in nonrobotic surgery, changes in distance during teleoperation phases are quite dramatic for the team. While team members usually gather in close, often intimate, physical contact, the robot redistributes them throughout the room at distances similar to those between co-workers in an office space.

Operating rooms are full of machines, which mediate practice by their sheer size or shape [77] and their specific interaction proxemics [48]: e.g. a computer screen outside the sterile field cannot be touched by sterile surgeons, leading them to ask nonsterile team members to manipulate images on the computer [54]. However, the robot is unique in its dramatic change of interaction proxemics. The distance increase evoked by the surgical teleoperation robot is a multitude of the common distance in nonrobotic and non-teleoperation phases and persists for large parts of the surgery. Translating this distance increase to an office setting, co-workers who used to share the same desk group would be moved to another floor or building. Distributing team members in space, the robot allows for more personal space and less intimate touch. At the same time, the robot largely prohibits nonverbal interaction, thereby shaping new interaction practices, such as uncovered by the cognitive and affective lenses of our framework. 


\subsection{Cognitive Distance}

Taking cognitive distance as a lens to study how the robot remediates teamwork in the operating room enabled us to understand changes in task-related practices as a result of physical changes Making use of our framework, we can not only describe how the robot mediates practice but also explain it as a result of the increased distance. As we have illustrated in sections 4.2.1 and 4.2.2, team members employ specific practices to build and maintain common ground during robotic surgery. In line with previous research $[20,50,60,72,86]$, we observed an increase in verbal communication in robotic surgery as compared to nonrobotic surgery. We observed that establishing situation awareness is a challenge for surgeons when in the role of the robot operator. This is in line with previous work on surgical robots [72,73] and literature on teleoperation [12, 21]. Further, difficulties with building and maintaining common ground in robotic surgery have been reported by others $[13,86]$. As reported also by Randell and colleagues [73], the surgical team successfully adjusts communication practice to mitigate challenges in grounding by employing readback, a strategy from airplane communication, to ensure that both sides have understood the information that has been verbally transmitted.

While previous work is largely in line with our findings, it has failed to provide comprehensive accounts of why team members adjust their practice. Applying our framework, lack of situation awareness and difficulties in establishing common ground can be understood as a result of changes in spatial arrangement. Establishing a focus on distance, relation to similar findings from collaboration over distance $[19,38,63]$ becomes possible. The increase in verbal communication is the result of practices that team members develop to address the lack of situation awareness and common ground as surgeons and team members need to ask for information explicitly (see 4.2.1 and 4.2.2). Thereby, our framework does not only shed light on how practice is mediated by a teleoperation robot but also provides insights on the underlying reasons of why this happens.

Previous work $[13,86]$ describes an increase in information flow, which it attributes to the fact that information on the state of the robot needs to be communicated. This is certainly true for some interactions (see e.g. Vignette 3), when trouble with (parts of) the robot occurs and needs to be dealt with. In the teams we observed, such trouble was largely fixed nonverbally after common ground on the problem was established. We would like to stress that more information than only on the state of the robot needs to be communicated explicitly in robotic surgery, as the surgeon is largely unaware of team member's actions, independently of their relation to the robot (see e.g. 4.2.1). The robot thus mediates communication on a more general level, as team members need to verbalize all information that is relevant for maintaining common ground on the surgery during the teleoperation phase. $[13,86]$ 's studies were done in the early days of robotic surgery and largely reflect the team's struggle to get accustomed to a new technology. As our study shows, findings may be more comprehensive when focusing on enacted practice [65] and studying how a technology mediates practice on a long-term basis.

Furthermore, focusing on the entire team, we gained a more thorough understanding of how teamwork is mediated by the robot. While previous work has described the lack of situation awareness for the surgeon, we would like to stress that this phenomenon occurs both ways and the team also sometimes lacks situation awareness of what the surgeon experiences (see 4.2.2, 4.2.3). The role of a lack of situation awareness for the team as a whole has largely been neglected by prior work. Our research extends understanding of situation awareness in teleoperation by showing that difficulties in situation awareness are not limited to robot operators but are an issue faced by both the operator and the team and mutual efforts are required to build shared situation awareness. 


\subsection{Affective Distance}

Changes in spatial distribution and specifically distance do not only affect task-related "cognitive" aspects, but also influence affective practice. As we have shown in section 4.3, team members are highly sensitive for each other's affective state and develop practices to deal with affective distance during robotic surgery. They increase the huddle, sometimes decreasing distance to zero: the nurses are touching the surgeon's head before the robot is entering the surgical field (see Figure 4). Our framework has proved crucial for this finding, as it highlights the close connection between spatial distance and affect. Puig de la Bellacasa [69] has pointed to the relation between physical proximity and affect: being in close proximity allows being in touch, physically and affectively. Understanding touch as a way to make sense of the world, touching someone may be understand as getting a feeling for how the other is doing. While this connection has been pointed out before, it has not been consequently applied to understand work practice. Our framework has helped to uncover the connection between being in close distance, which allows touch, and being in affective touch with each other. The team's huddle in robotic surgery then may be understood as a practice to make sense of the team member's affective state. As touching someone inevitably implies being touched as well [69] the huddle can be understood as a practice for mutual affective sensemaking. This fits well with the notion of "affective grounding" put forward by Jung [40], who proposes that participants of an interaction not only have to coordinate on process and content but also on affect, developing a shared understanding of the affective dimension of their interaction. It is important for team members to coordinate on their affective state in order to maintain close collaboration. In this sense, our work extends the understanding of affective grounding by showing how the huddle of a team is a practice to build and maintain affective ground in a high-stress work environment. Focusing on the close connection between proximity and affect, our framework has helped to uncover previously disregarded aspects of surgical work. Hirschauer [35] claims that team members suppress expressions of emotion. While we have indeed not observed emotional outbursts (e.g. someone shouting in anger), we did identify moments when the affective awareness and the coordination of the team's affective state became relevant, such as illustrated in Vignette 4. As affective ground is negotiated largely nonverbally and communicated through touch or close distance, it seems to have been easily missed by other analytic approaches.

\subsection{Implications and Future Work}

Reconfiguration of teamwork into a form of collaboration that shares characteristics of collocated and remote teamwork has several implications for our understanding of tele-robot supported surgery and teamwork. First, there are implications for the distribution of power within surgical teams: In contrast to [38], the surgeon as a leader is not tasked with cultivating a global understanding of what is happening in the operating room and coordinating action but rather has to carry out his or her own task and the entire team has to work on maintaining common ground and coordinating actions. When the surgeon as the leader of the team is remote in the role of the robot operator, the power structure is affected, as team members who have little influence suddenly gain in autonomy, as the surgeon is dependent on them to give appropriate feedback or carrying out actions by the patient-side which the surgeon cannot oversee. This is in line with previous work, which suggests that the role of the team is enhanced $[72,73]$ as the surgeon is dependent on the team to communicate crucial information [50] and to carry out additional tasks $[20,50]$. The robot thus changes power relations, as everyone is mutually dependent on each other for exchanging information and maintaining common ground. If the head of the team is removed to a more remote site and not visually present any more, this may change power relations as new dependencies are created. At the same time, the design of the robotic surgical systems re-enforces existing power 
structures, as it centers task load on the surgeon $[2,73]$, neglecting the relevance of the other team members or even taking over their tasks [85]. The use of the robot facilitates a power structure that stresses the role of the surgeon as the most important member of the team while deemphasizing the importance of other team members. While the literature has been divided on this, we would like to stress that this duality seems to be inherent and characteristic of this work. Focusing on the surgeon as the main actor in the role of the robot operator, one may easily overlook the team, yet, without the team the entire surgery would be impossible, as the surgeon would constantly have to move back and forth between patient-side and robot console, scrubbing and unscrubbing as he or she enters and leaves the sterile field. Recent work has shown that conflict in surgical teams is often initiated by team members who rank high in the hierarchy, such as the surgeon [39]. While [39] studied open and laparoscopic surgeries, they did not include robotic surgeries in their analysis. It would be interesting to investigate in future research whether the shifts in power dynamics in robotic surgery also have an impact on conflict.

Second, we have shown the importance of sensitivity to individual and collective affective states in maintaining more positive and collaborative working relations. Tele-robot assisted surgery as practiced in the settings we studied relies on increased engagement in affective grounding during collocated phases, thereby making up for the reduced sensitivity during distributed teleoperation phases. As the importance of regular intensive face-to-face interaction has also been pointed out for effective globally distributed teams [53], we think this is a crucial practice for distributed teamwork and teleoperation in general, independent of the amount of distance between team members. Taken more broadly, and consistent with recent scholarship in science and technology studies [36, 59, 68, 70,71], this finding connects questions of distance and mediation to the ongoing centrality of care work in the relationships and practices of teams, (reminding us that surgical teams not only perform but also in some measure run on care). The affective implications of the introduction of robots into collaborative work settings like the one studied here deserve further and careful study, and should be brought more squarely into the design, deployment, and creative organizational adoption of emerging robotic technologies.

Third, the framework we have developed for studying how technology mediates teamwork and practice in a close-knit team may also be useful for studying technologies other than teleoperation robots. Krogh and colleagues [48] have shown that displays of different sizes have different interaction proxemics, affording users to spatially arrange themselves around a display with respect to its size. As we have demonstrated how spatial reconfiguration may influence cognitive and affective aspects of teamwork, it is likely that such different spatial configurations afforded by a specific technology may also bring about differences in a team's cognitive and affective practices. This should be investigated further, as it may help to uncover how technology affects collaborative practice and sensemaking - not only with respect to task-related aspects but also in terms of interpersonal affect. It could be especially interesting to apply our framework in telepresence settings, as spatial positioning and interpersonal distance are constrained by the telepresence technology. Scrutinizing how different spatial arrangements and distance afforded by telepresence devices (e.g. team member tele-present on a hand-held smartphone vs. via a telepresence robot that physically joins the team vs. on the distant screen of a conference room) mediate cognitive and affective aspects of teamwork could yield interesting insights. Finally, our physical-cognitive-affective distance framework is not only useful for the study of how existing technology mediates teamwork but also relevant for designers of collaboration technology. The insight that different physical distances facilitate or inhibit certain cognitive and affective practices may be used to purposefully evoke certain practices by building particular interaction proxemics into a collaboration technology, e.g. forcing team members to huddle or spread in order to accomplish their tasks. Designing with distance in mind 
may thus allow to gently nudge teams towards effective collaboration practices in cognitive and affective terms.

\section{CONCLUSION}

In this paper we have investigated how team practices are reconfigured when an existing team member, the leader, takes on the role of the operator for a telepresence robot and is thereby placed at a distance from the team. Analyzing changes along physical, cognitive and affective dimensions, we have shown how the robot ultimately reconfigures collaborative practices within the team in ways that show characteristics and challenges of both collocated and distributed work.

\section{ACKNOWLEDGMENTS}

We thank all participants for giving us invaluable insights into their work. Special thanks to the participating surgeons for allowing us to shadow them and to the hospital administrations for granting us access to their operating rooms. We would like to express our gratitude to the anonymous reviewers for providing helpful comments on previous versions of this document. Dr. Mariët Theune deserves our thanks for giving feedback on an earlier version of the manuscript. This material is based upon work supported by Cornell University's Institute for the Social Sciences and by the National Science Foundation under Grant No. 1563705. Any opinions, findings, and conclusions or recommendations expressed in this material are those of the authors and do not necessarily reflect the views of the National Science Foundation.

\section{REFERENCES}

[1] Hoorieh Afkari, Roman Bednarik, Susanne Mäkelä, and Shahram Eivazi. 2016. Mechanisms for maintaining situation awareness in the micro-neurosurgical operating room. International fournal of Human-Computer Studies 95 (2016), $1-14$.

[2] Natasha Alvarado, Stephanie Honey, Joanne Greenhalgh, Alan Pearman, Dawn Dowding, Alexandra Cope, Andrew Long, David Jayne, Arron Gill, Alwyn Kotze, et al. 2017. Eliciting context-mechanism-outcome configurations: Experiences from a realist evaluation investigating the impact of robotic surgery on teamwork in the operating theatre Evaluation 23, 4 (2017), 444-462.

[3] Peter M. Asaro. 2013. The labor of surveillance and bureaucratized killing: New subjectivities of military drone operators. Social Semiotics 23, 2 (2013), 196-224. https://doi.org/10.1080/10350330.2013.777591

[4] Jakob E. Bardram. 2000. Temporal coordination - On time and coordination of collaborative activities at a surgical department. Computer Supported Cooperative Work (CSCW) 9, 2 (2000), 157-187.

[5] Jakob E. Bardram and Claus Bossen. 2005. Mobility work: The spatial dimension of collaboration at a hospital. Computer Supported Cooperative Work (CSCW) 14, 2 (April 2005), 131-160. https://doi.org/10.1007/s10606-005-0989-y

[6] Jakob E. Bardram and Thomas Riisgaard Hansen. 2010. Why the plan doesn't hold: A study of situated planning, articulation and coordination work in a surgical ward. In Proceedings of the 2010 ACM Conference on Computer Supported Cooperative Work (CSCW'10). ACM, New York, NY, USA, 331-340. https://doi.org/10.1145/1718918.1718977

[7] Matthew Beane. 2018. Shadow learning: Building robotic surgical skill when approved means fail. Administrative Science Quarterly (2018), 0001839217751692.

[8] Jeff Bezemer, Ged Murtagh, Alexandra Cope, Gunther Kress, and Roger Kneebone. 2011. "Scissors, please": The practical accomplishment of surgical work in the operating theater. Symbolic Interaction 34, 3 (2011), 398-414.

[9] Pernille Bjørn, Morten Esbensen, Rasmus Eskild Jensen, and Stina Matthiesen. 2014. Does distance still matter? Revisiting the CSCW fundamentals on distributed collaboration. ACM Transactions on Computer-Human Interaction (TOCHI) 21, 5, Article 27 (Nov. 2014), 26 pages. https://doi.org/10.1145/2670534

[10] John F. Boggess. 2007. Robotic surgery in gynecologic oncology: Evolution of a new surgical paradigm. fournal of Robotic Surgery 1, 1 (2007), 31-37.

[11] Judee K. Burgoon. 2015. Expectancy violations theory. The International Encyclopedia of Interpersonal Communication (2015). https://doi.org/10.1002/9781118540190.wbeic102

[12] Jennifer L. Burke, Robin R. Murphy, Michael D. Coovert, and Dawn L. Riddle. 2004. Moonlight in Miami: Field study of human-robot interaction in the context of an urban search and rescue disaster response training exercise. Human-Computer Interaction 19, 1-2 (2004), 85-116. 
[13] Caroline G. Cao and Holly Taylor. 2004. Effects of new technology on the operating room team. In Proceedings of the 7th International Conference on Work with Computing Systems. Damai Sciences, 309-312. http://www.dtic.mil/dtic/tr/ fulltext/u2/a438402.pdf

[14] Julie Carpenter. 2016. Culture and human-robot interaction in militarized spaces: A war story. Routledge, London.

[15] Jennifer Casper and Robin R. Murphy. 2003. Human-robot interactions during the robot-assisted urban search and rescue response at the world trade center. IEEE Transactions on Systems, Man, and Cybernetics, Part B (Cybernetics) 33 , 3 (2003), 367-385.

[16] Kathy Charmaz. 2006. Constructing grounded theory: A practical guide through qualitative analysis. Sage, London.

[17] Herbert H. Clark. 1996. Using language. Cambridge University Press, Cambridge.

[18] Amelia Compagni, Valentina Mele, and Davide Ravasi. 2015. How early implementations influence later adoptions of innovation: Social positioning and skill reproduction in the diffusion of robotic surgery. Academy of Management Journal 58, 1 (2015), 242-278.

[19] Catherine D. Cramton. 2001. The mutual knowledge problem and its consequences for dispersed collaboration. Organization Science 12, 3 (2001), 346-371.

[20] Stacey Cunningham, Amine Chellali, Isabelle Jaffre, J. Classe, and Caroline G. Cao. 2013. Effects of experience and workplace culture in human-robot team interaction in robotic surgery: A case study. International fournal of Social Robotics 5, 1 (2013), 75-88.

[21] Jill L. Drury, Jean Scholtz, and Holly A. Yanco. 2003. Awareness in human-robot interactions. In Proceedings of the 2003 IEEE International Conference on Systems, Man and Cybernetics, Vol. 1. IEEE, 912-918.

[22] Edeltraud Egger and Ina Wagner. 1992. Negotiating temporal orders. Computer Supported Cooperative Work (CSCW) 1 , 4 (1992), 255-275.

[23] Robert M. Emerson, Rachel I. Fretz, and Linda L. Shaw. 2011. Writing ethnographic fieldnotes. University of Chicago Press, Chicago, IL.

[24] Mica R. Endsley. 1995. Toward a theory of situation awareness in dynamic systems. Human Factors 37, 1 (1995), 32-64.

[25] Terrence Fong and Charles Thorpe. 2001. Vehicle teleoperation interfaces. Autonomous Robots 11, 1 (July 2001), 9-18. https://doi.org/10.1023/A:1011295826834

[26] Rajiv B. Gala, Rebecca Margulies, Adam Steinberg, Miles Murphy, James Lukban, Peter Jeppson, Sarit Aschkenazi, Cedric Olivera, Mary South, Lior Lowenstein, et al. 2014. Systematic review of robotic surgery in gynecology: Robotic techniques compared with laparoscopy and laparotomy. fournal of Minimally Invasive Gynecology 21, 3 (2014), 353-361.

[27] Kristina Groth and Jeremiah Scholl. 2013. Coordination in highly-specialized care networks. In Proceedings of the 2013 Conference on Computer Supported Cooperative Work Companion (CSCW '13). ACM, New York, NY, USA, 143-148. https://doi.org/10.1145/2441955.2441992

[28] Carsten N. Gutt, T. Oniu, Arianeb Mehrabi, A. Kashfi, P. Schemmer, and Markus W. Büchler. 2004. Robot-assisted abdominal surgery. British fournal of Surgery 91 (2004), 1390-1397. https://doi.org/10.1002/bjs.4700

[29] Edward T. Hall. 1966. The hidden dimension. Doubleday, Garden City, NY.

[30] Christian Heath, Jon Hindmarsh, and Paul Luff. 2010. Video in qualitative research. Sage Publications, London.

[31] Christian Heath, Marcus Sanchez Svensson, Jon Hindmarsh, Paul Luff, and Dirk Vom Lehn. 2002. Configuring awareness. Computer Supported Cooperative Work (CSCW) 11, 3-4 (2002), 317-347.

[32] Jon Hindmarsh and Alison Pilnick. 2002. The tacit order of teamwork: Collaboration and embodied conduct in anesthesia. The Sociological Quarterly 43, 2 (2002), 139-164.

[33] Jon Hindmarsh and Alison Pilnick. 2007. Knowing bodies at work: Embodiment and ephemeral teamwork in anaesthesia. Organization Studies 28, 9 (2007), 1395-1416.

[34] Pamela J. Hinds and Mark Mortensen. 2005. Understanding conflict in geographically distributed teams: The moderating effects of shared identity, shared context, and spontaneous communication. Organization Science 16, 3 (2005), $290-307$.

[35] Stefan Hirschauer. 1991. The manufacture of bodies in surgery. Social Studies of Science 21, 2 (1991), 279-319.

[36] Carla Hustak and Natasha Myers. 2012. Involutionary momentum: Affective ecologies and the sciences of plant/insect encounters. Differences 23, 3 (2012), 74-118.

[37] IntuitiveSurgical. 2018. Intuitive Surgical Announces Preliminary Fourth Quarter and Full Year 2017 Results [Press release]. (January 10 2018). Retrieved September 2, 2018 from http://isrg.gcs-web.com/press-releases

[38] Hank Jones and Pamela Hinds. 2002. Extreme work teams: Using SWAT teams as a model for coordinating distributed robots. In Proceedings of the 2002 ACM Conference on Computer Supported Cooperative Work (CSCW '02). ACM, New York, NY, USA, 372-381. https://doi.org/10.1145/587078.587130

[39] Laura K. Jones, Bonnie Mowinski Jennings, Melinda K. Higgins, and Frans B. M. de Waal. 2018. Ethological observations of social behavior in the operating room. Proceedings of the National Academy of Sciences (2018). https://doi.org/10 1073/pnas.1716883115

[40] Malte F. Jung. 2017. Affective grounding in human-robot interaction. In Proceedings of the 2017 ACM/IEEE International Conference on Human-Robot Interaction (HRI '17). ACM, New York, NY, USA, 263-273. https://doi.org/10.1145/2909824. 
3020224

[41] Adam Kendon. 2010. Spacing and orientation in co-present interaction. In Proceedings of the Second International Conference on Development of Multimodal Interfaces: Active Listening and Synchrony (COST'09). Springer, Berlin, Heidelberg, 1-15. https://doi.org/10.1007/978-3-642-12397-9_1

[42] Young Tae Kim, Sang Wun Kim, and Yong Wook Jung. 2008. Robotic surgery in gynecologic field. Yonsei Medical fournal 49, 6 (2008), 886-890.

[43] Timothy Koschmann, Curtis LeBaron, Charles Goodwin, and Paul Feltovich. 2006. The mystery of the missing referent: Objects, procedures, and the problem of the instruction follower. In Proceedings of the 200620 th Anniversary Conference on Computer Supported Cooperative Work (CSCW'06). ACM, New York, NY, USA, 373-382. https://doi.org/10.1145/ 1180875.1180932

[44] Timothy Koschmann, Curtis LeBaron, Charles Goodwin, and Paul Feltovich. 2011. "Can you see the cystic artery yet?" A simple matter of trust. Journal of Pragmatics 43, 2 (2011), 521-541.

[45] Timothy Koschmann, Curtis LeBaron, Charles Goodwin, Alan Zemel, and Gary Dunnington. 2007. Formulating the triangle of doom. Gesture 7, 1 (2007), 97-118.

[46] Timothy Koschmann and Alan Zemel. 2011. So that's the ureter: The informal logic of discovering work. Ethnographic Studies 12 (2011), 31-46.

[47] Robert E. Kraut, Susan R. Fussell, Susan E. Brennan, and Jane Siegel. 2002. Understanding effects of proximity on collaboration: Implications for technologies to support remote collaborative work. Distributed Work (2002), 137-162.

[48] Peter Gall Krogh, Marianne Graves Petersen, Kenton O’Hara, and Jens Emil Groenbaek. 2017. Sensitizing concepts for socio-spatial literacy in HCI. In Proceedings of the 2017 CHI Conference on Human Factors in Computing Systems (CHI '17). ACM, New York, NY, USA, 6449-6460. https://doi.org/10.1145/3025453.3025756

[49] Daniel Labonte, Patrick Boissy, and François Michaud. 2010. Comparative analysis of 3-D robot teleoperation interfaces with novice users. IEEE Transactions on Systems, Man, and Cybernetics, Part B (Cybernetics) 40, 5 (2010), 1331-1342.

[50] Fuji Lai and Eileen Entin. 2005. Robotic surgery and the operating room team. In Proceedings of the Human Factors and Ergonomics Society Annual Meeting, Vol. 49. Sage Publications, Los Angeles, CA, 1070-1073.

[51] Gyusung I. Lee, Mija R. Lee, Tamera Clanton, Erica Sutton, Adrian E. Park, and Michael R. Marohn. 2014. Comparative assessment of physical and cognitive ergonomics associated with robotic and traditional laparoscopic surgeries. Surgical Endoscopy 28, 2 (2014), 456-465.

[52] Sergio Maeso, Mercedes Reza, Julio A. Mayol, Juan A. Blasco, Mercedes Guerra, Elena Andradas, and María N. Plana. 2010. Efficacy of the da vinci surgical system in abdominal surgery compared with that of laparoscopy: A systematic review and meta-analysis. Annals of Surgery 252, 2 (2010), 254-262.

[53] Martha L. Maznevski and Katherine M. Chudoba. 2000. Bridging space over time: Global virtual team dynamics and effectiveness. Organization Science 11, 5 (2000), 473-492.

[54] Helena M. Mentis, Kenton O’Hara, Abigail Sellen, and Rikin Trivedi. 2012. Interaction proxemics and image use in neurosurgery. In Proceedings of the SIGCHI Conference on Human Factors in Computing Systems (CHI '12). ACM, New York, NY, USA, 927-936. https://doi.org/10.1145/2207676.2208536

[55] Helena M. Mentis and Alex S. Taylor. 2013. Imaging the body: Embodied vision in minimally invasive surgery. In Proceedings of the SIGCHI Conference on Human Factors in Computing Systems (CHI '13). ACM, New York, NY, USA, 1479-1488. https://doi.org/10.1145/2470654.2466197

[56] Natasha Merat, Hamish A. Jamson, Frank Lai, and Oliver Carsten. 2014. Human factors of highly automated driving: Results from the EASY and CityMobil projects. In Road Vehicle Automation. Lecture Notes in Mobility. Springer, Cham, 113-125.

[57] Lorenza Mondada. 2014. Instructions in the operating room: How the surgeon directs their assistant's hands. Discourse Studies 16, 2 (2014), 131-161.

[58] Robin R. Murphy and Jennifer L. Burke. 2008. From remote tool to shared roles. IEEE Robotics \& Automation Magazine 15, 4 (2008).

[59] Natasha Myers. 2008. Molecular embodiments and the body-work of modeling in protein crystallography. Social Studies of Science 38, 2 (2008), 163-199.

[60] Anne-Sophie Nyssen and Adelaïde Blavier. 2009. Verbal communication as a sign of adaptation in socio-technical systems: The case of robotic surgery. In Proceedings of the 9th International Conference on Naturalistic Decision Making (NDM9,). British Computer Society, London, 267-272.

[61] Kenton O’Hara, Jesper Kjeldskov, and Jeni Paay. 2011. Blended interaction spaces for distributed team collaboration. ACM Transactions on Computer-Human Interaction (TOCHI) 18, 1, Article 3 (May 2011), 28 pages. https://doi.org/10. $1145 / 1959022.1959025$

[62] Allison M. Okamura, Maja J. Mataric, and Henrik I. Christensen. 2010. Medical and health-care robotics. IEEE Robotics \& Automation Magazine 17, 3 (2010), 26-37.

[63] Gary M. Olson and Judith S. Olson. 2000. Distance matters. Human-Computer Interaction 15, 2 (2000), $139-178$. 
[64] Mark J. V. Olson. 2009. Robotic surgery, human fallibility, and the politics of care. Ph.D. Dissertation. The University of North Carolina at Chapel Hill.

[65] Wanda J. Orlikowski. 2008. Using technology and constituting structures: A practice lens for studying technology in organizations. In Resources, Co-Evolution and Artifacts. Springer, London, 255-305. https://doi.org/10.1007/ 978-1-84628-901-9_10

[66] Resad P. Pasic, John A. Rizzo, Hai Fang, Susan Ross, Matt Moore, and Candace Gunnarsson. 2010. Comparing robotassisted with conventional laparoscopic hysterectomy: Impact on cost and clinical outcomes. Fournal of Minimally Invasive Gynecology 17, 6 (2010), 730-738. https://doi.org/10.1016/j.jmig.2010.06.009

[67] Thomas N. Payne and Francis R. Dauterive. 2008. A comparison of total laparoscopic hysterectomy to robotically assisted hysterectomy: Surgical outcomes in a community practice. fournal of Minimally Invasive Gynecology 15, 3 (2008), 286-291. https://doi.org/10.1016/j.jmig.2008.01.008

[68] Rachel Prentice. 2012. Bodies in formation: Remaking anatomy and surgery education. Duke University Press, Durham, NC.

[69] María Puig de la Bellacasa. 2009. Touching technologies, touching visions. The reclaiming of sensorial experience and the politics of speculative thinking. Subjectivity 28, 1 (01 Sep 2009), 297-315. https://doi.org/10.1057/sub.2009.17

[70] María Puig de la Bellacasa. 2011. Matters of care in technoscience: Assembling neglected things. Social Studies of Science 41, 1 (2011), 85-106. https://doi.org/10.1177/0306312710380301

[71] María Puig de La Bellacasa. 2017. Matters of care. Speculative ethics in more than human worlds. University of Minnesota Press, Minneapolis, MN.

[72] Rebecca Randell, Natasha Alvarado, Stephanie Honey, Joanne Greenhalgh, Peter Gardner, Arron Gill, David Jayne, Alwyn Kotze, Alan Pearman, and Dawn Dowding. 2015. Impact of robotic surgery on decision making: Perspectives of surgical teams. In AMIA Annual Symposium Proceedings, Vol. 2015. American Medical Informatics Association, 1057-1066.

[73] Rebecca Randell, Stephanie Honey, Natasha Alvarado, Alan Pearman, Joanne Greenhalgh, Andrew Long, Peter Gardner, Arron Gill, David Jayne, and Dawn Dowding. 2016. Embedding robotic surgery into routine practice and impacts on communication and decision making: A review of the experience of surgical teams. Cognition, Technology \& Work 18 , 2 (2016), 423-437.

[74] Rebecca Randell, Stephanie Honey, Jon Hindmarsh, Natasha Alvarado, Joanne Greenhalgh, Alan Pearman, Andrew Long, Alexandra Cope, Arron Gill, Peter Gardner, et al. 2017. A realist process evaluation of robot-assisted surgery: Integration into routine practice and impacts on communication, collaboration and decision-making. Health Services and Delivery Research 5, 20 (Jun 2017), 1-140. https://doi.org/10.3310/hsdr05200

[75] Lauren Schiff, Ziv Tsafrir, Joelle Aoun, Andrew Taylor, Evan Theoharis, and David Eisenstein. 2016. Quality of communication in robotic surgery and surgical outcomes. Fournal of the Society of Laparoendoscopic Surgeons 20, 3 (2016).

[76] Solace Shen, Hamish Tennent, Houston Claure, and Malte Jung. 2018. My telepresence, my culture?: An intercultural investigation of telepresence robot operators' interpersonal distance behaviors. In Proceedings of the 2018 CHI Conference on Human Factors in Computing Systems (CHI '18). ACM, New York, NY, USA, Article 51, 11 pages. https://doi.org/10 $1145 / 3173574.3173625$

[77] Robert Sommer and Hugo Ross. 1958. Social interaction on a geriatrics ward. International fournal of Social Psychiatry 4, 2 (1958), 128-133.

[78] Brett Stoll, Samantha Reig, Lucy He, Ian Kaplan, Malte F. Jung, and Susan R. Fussell. 2018. Wait, can you move the robot? Examining telepresence robot use in collaborative teams. In Proceedings of the 2018 ACM/IEEE International Conference on Human-Robot Interaction (HRI '18). ACM, New York, NY, USA, 14-22. https://doi.org/10.1145/3171221.3171243

[79] Anselm L. Strauss and Juliet M. Corbin. 1998. Basics of qualitative research: Techniques and procedures for developing grounded theory. Sage Publications, Thousand Oaks, CA.

[80] Marcus Sanchez Svensson, Christian Heath, and Paul Luff. 2007. Instrumental action: The timely exchange of implements during surgical operations. In Proceedings of the 10th European Conference on Computer Supported Cooperative Work (ECSCW 2007). Springer, 41-60.

[81] Marcus Sanchez Svensson, Paul Luff, and Christian Heath. 2009. Embedding instruction in practice: Contingency and collaboration during surgical training. Sociology of Health \& Illness 31, 6 (2009), 889-906.

[82] Leila Takayama and Caroline Pantofaru. 2009. Influences on proxemic behaviors in human-robot interaction. In Proceedings of the IEEE/RSF International Conference on Intelligent Robots and Systems (IROS 2009). IEEE, 5495-5502.

[83] Janet Vertesi. 2015. Seeing like a rover: How robots, teams, and images craft knowledge of mars. University of Chicago Press, Chicago, IL.

[84] Jered Vroon, Michiel Joosse, Manja Lohse, Jan Kolkmeier, Jaebok Kim, Khiet Truong, Gwenn Englebienne, Dirk Heylen, and Vanessa Evers. 2015. Dynamics of social positioning patterns in group-robot interactions. In 24th IEEE International Symposium on Robot and Human Interactive Communication (RO-MAN 2015). IEEE, 394-399. https: 
//doi.org/10.1109/ROMAN.2015.7333633

[85] Kristian Wasen. 2010. Replacement of highly educated surgical assistants by robot technology in working life: Paradigm shift in the service sector. International fournal of Social Robotics 2, 4 (2010), 431-438.

[86] Jessica L. Webster and Caroline G. L. Cao. 2006. Lowering communication barriers in operating room technology. Human Factors 48, 4 (2006), 747-758.

[87] John R. Wilson, Ann Mills, Theresa Clarke, Jane Rajan, and Nastaran Dadashi. 2012. Rail human factors around the world: Impacts on and of people for successful rail operations. CRC Press, Leiden, The Netherlands.

[88] Robert N. Wilson. 1954. Teamwork in the operating room. Human Organization 12, 4 (1954), 9-14.

Received April 2018; revised July 2018; accepted September 2018 